\title{
Application of Opposition-Based Learning Concepts in Reducing the Power Consumption in Wireless Access Networks
}

\author{
Sotirios K. Goudos \\ Department of Physics \\ Aristotle University of Thessaloniki \\ Thessaloniki 54124, Greece \\ Email: sgoudo@physics.auth.gr
}

\author{
Margot Deruyck, David Plets, Luc Martens, \\ Wout Joseph \\ Department of Information Technology \\ Ghent University \\ Ghent, Belgium \\ Email: \{margot.deruyck, david.plets, \\ luc.martens, wout.joseph $\} @$ intec.ugent.be
}

\begin{abstract}
The reduction of power consumption in wireless access networks is a challenging and important issue. In this paper, we apply Opposition-Based Learning (OBL) concepts for reducing the power consumption of LTE base stations. More specifically, we present a new Modified Biogeography Based Optimization (BBO) algorithm enhanced with OBL techniques. We apply both the original BBO and the new Modified Opposition BBO (MOBBO) to network design cases to the city of Ghent, Belgium, with 75 possible LTE base station locations. We optimize the network towards two objectives: coverage maximization and power consumption minimization. Preliminary results indicate the advantages and applicability of our approach.
\end{abstract}

\section{INTRODUCTION}

Wireless access networks are currently large power consumers within ICT. In five years time (from 2007 till 2012), this power consumption has increased yearly with $10 \%$ [1]. It is expected that this amount will even increase in the next few years, as these networks need to expand in order to deal with the extreme growth of mobile devices and the higher bit rate demands required by these mobile devices. For the development of future wireless access networks, power consumption will become a key parameter [2]-[5]. A specified area, the target area, needs to be covered with a certain wireless technology. In this paper we consider Long Term Evolution (LTE) with a minimal power consumption. By selecting the most appropriate base station locations from a set of existing locations (from operators active in the target area) and tuning base station parameters such as the antennas input power, an energy-efficient network is obtained. Additionally, we optimize the network by taking into account Multiple Input Multiple Output (MIMO) for each base station and assuming that each base station could be either a macrocell or a femtocell. Evolutionary algorithms (EAs) are suitable optimization techniques for solving the above-described problem.

Biogeography-based optimization (BBO) [6] is an evolutionary algorithm based on mathematical models that describe how species migrate from one island to another, how new species arise, and how species become extinct. The way the problem solution is found is analogous to natures way of distributing species. In [7] a new BBO algorithm based on opposition-based learning (OBL) called Oppositional Biogeography-Based Optimization (OBBO) was introduced. The basic idea of the OBL concept is to calculate the fitness not only of the current individual but also to calculate the fitness of the opposite individual. The benefits of using such a technique are that convergence speed may be faster and that a better approximation of the global optimum can be found. OBL techniques were also applied successfully to Differential Evolution in [8]. In all the above papers, OBL was applied to continuous domain problems. In [9] the OBBO concept was applied to specific discrete domain problems like the traveling salesman (TSP) and the vertex coloring problem. However, in the above paper the definition of the opposite point was problem-dependent.

In this paper we propose a new Modified Opposition BBO (MOBBO) that can be applied to the network design problems and to other discrete domain problems as well. The basic concept of the proposed algorithm is to decide using a predefined opposition probability if each decision variable in every Ddimensional individual is replaced by its opposite or not.

We apply MOBBO to three design cases for power reduction and coverage maximization for LTE networks. We compare results with the original $\mathrm{BBO}$. Numerical results show that MOBBO outperforms the original BBO algorithm in terms of solution accuracy and convergence speed. This paper is organized as follows. We describe the problem formulation in Section II. The details of the MOBBO algorithm are given in Section III. In Section IV we present the numerical results. Finally, the conclusion is given in Section V.

\section{FORMULATION}

We address network planning optimization for LTE base stations. The concept and algorithms are used to perform network planning of 75 LTE base station locations in the city of Ghent, Belgium. This area covers about $6.85 \mathrm{~km}^{2}$. The network optimization problem is to find the least possible number of base stations that operate with such input power 
so that the coverage area is maximized. Therefore, there are two requirements; to minimize power consumption and to maximize coverage. The power consumption objective can be expressed as [2]:

$$
f_{\text {pow }}(\bar{x})=100\left(1-\frac{P_{c}(\bar{x})}{P_{\max }}\right)
$$

where $\bar{x}$ is the vector of a given solution, $P_{c}(\bar{x})$ is the calculated power consumption in Watts of the solution, and $P_{\max }$ is the maximum power consumption assuming that all base stations are active and operate at maximum input power. In case of a femtocell base station we consider a fixed power consumption of $12 \mathrm{~W}$. The details of the power consumption formulation can be found in [2]. The second objective is to cover the maximum possible percentage of the given area. The coverage function $f_{\text {cov }}(\bar{x})$ specified by:

$$
f_{\text {cov }}(\bar{x})=100 \frac{A_{\text {target }} \bigcap A(\bar{x})}{A_{\text {target }}}
$$

where $A_{\text {target }}$ is the area of the target area to be covered (in $\mathrm{km}^{2}$ ), and $A(\bar{x})$ is the area covered by a given solution (in $\mathrm{km}^{2}$ ). In order to calculate the $A(\bar{x})$ we first needed to calculate for each active base station the maximum allowable path loss, PLmax (in dB). For this case, the link budget parameters for the LTE network of Table I are taken into account. The maximum range $R$ (in meters) covered by each base station can be computed as in [2]. The area covered by a given solution is the union of all base stations coverage areas that are determined by each maximum range $R$. The above objectives can be combined using the following objective function [2]:

$$
\begin{aligned}
& F(\bar{x})=-\left(f_{\text {cov }}(\bar{x})+k f_{\text {pow }}(\bar{x})\right) \\
& \text { with } \\
& k=\left\{\begin{array}{l}
0 \text { if } f_{\operatorname{cov}}(\bar{x})<90 \\
\frac{\left(f_{\operatorname{cov}}(\bar{x})-90\right)^{2}}{5} \text { if } 90 \leq f_{\operatorname{cov}}(\bar{x}) \geq 95 \\
5 \text { otherwise }
\end{array}\right.
\end{aligned}
$$

where the minus sign is used for minimization. The minimum value $(-600)$ is obtained when both $f_{\text {cov }}(\bar{x})$ and $f_{\text {pow }}(\bar{x})$ equal to 100. This kind of global fitness function is chosen because of the trade-off between coverage and power consumption.

In this paper, we assume that all femtocell base stations are placed outdoor. Additionally, we consider the WalfischIkegami propagation model for path loss calculations. The above-mentioned problem can be solved using an evolutionary algorithm. It is an integer-programming problem, for which several different solutions exist. In this paper, we will apply the BBO and the MOBBO algorithms.

\section{Modified OpPositionAL BIOGEOGRAPHY-BASED OPTIMIZATION}

The mathematical models of Biogeography are based on the

\begin{tabular}{|c|c|c|}
\hline Parameter & Macrocell BS & Femtocell BS \\
\hline Frequency & $2.6 \mathrm{GHz}$ & $2.6 \mathrm{GHz}$ \\
\hline $\begin{array}{l}\text { Maximum input } \\
\text { power base } \\
\text { antenna }\end{array}$ & $43 \mathrm{dBm}$ & $33 \mathrm{dBm}$ \\
\hline $\begin{array}{l}\text { Antenna gain of base } \\
\text { station }\end{array}$ & $18 \mathrm{dBi}$ & $4 \mathrm{dBi}$ \\
\hline $\begin{array}{l}\text { Antenna gain of re- } \\
\text { ceiver }\end{array}$ & $0 \mathrm{dBi}$ & $0 \mathrm{dBi}$ \\
\hline $\begin{array}{l}\text { Feeder loss base sta- } \\
\text { tion }\end{array}$ & $2 \mathrm{~dB}$ & $2 \mathrm{~dB}$ \\
\hline Feeder loss receiver & $0 \mathrm{~dB}$ & $0 \mathrm{~dB}$ \\
\hline Fade margin & $10 \mathrm{~dB}$ & $10 \mathrm{~dB}$ \\
\hline Yearly availability & $100.00 \%$ & $100.00 \%$ \\
\hline Interference margin & $2 \mathrm{~dB}$ & $2 \mathrm{~dB}$ \\
\hline $\begin{array}{l}\text { Noise figure of re- } \\
\text { ceiver }\end{array}$ & $8 \mathrm{~dB}$ & $8 \mathrm{~dB}$ \\
\hline $\begin{array}{l}\text { Implementation loss } \\
\text { of receiver }\end{array}$ & $0 \mathrm{~dB}$ & $0 \mathrm{~dB}$ \\
\hline MIMO & $1 \times 1$ & $1 \mathrm{x} 1$ \\
\hline Receiver SNR & $\begin{array}{l}1 / 3 \text { QPSK }=-1.5 \mathrm{~dB} \\
1 / 2 \text { QPSK }=3 \mathrm{~dB} \\
2 / 3 \text { QPSK }=10.5 \mathrm{~dB} \\
1 / 216-\mathrm{QAM}=14 \mathrm{~dB} \\
2 / 316-\mathrm{QAM}=19 \mathrm{~dB} \\
1 / 264-\mathrm{QAM}=23 \mathrm{~dB} \\
2 / 3 \quad 64-\mathrm{QAM}=29.4 \\
\mathrm{~dB}\end{array}$ & $\begin{array}{l}1 / 3 \mathrm{QPSK}=-1.5 \mathrm{~dB} \\
1 / 2 \mathrm{QPSK}=3 \mathrm{~dB} \\
2 / 3 \text { QPSK }=10.5 \mathrm{~dB} \\
1 / 216-\mathrm{Q} \mathrm{AM}=14 \mathrm{~dB} \\
2 / 316-\mathrm{Q} \mathrm{AM}=19 \mathrm{~dB} \\
1 / 264-\mathrm{Q} \mathrm{AM}=23 \mathrm{~dB} \\
2 / 364-\mathrm{QAM}=29.4 \\
\mathrm{~dB}\end{array}$ \\
\hline Bandwidth & $5 \mathrm{MHz}$ & $5 \mathrm{MHz}$ \\
\hline $\begin{array}{l}\text { Soft handover gain } \\
\text { receiver }\end{array}$ & $0 \mathrm{~dB}$ & $0 \mathrm{~dB}$ \\
\hline $\begin{array}{l}\text { Building penetration } \\
\text { loss }\end{array}$ & $\begin{array}{l}0 \mathrm{~dB} \text { (only outdoor } \\
\text { coverage considered) }\end{array}$ & $\begin{array}{l}0 \mathrm{~dB} \text { (only outdoor } \\
\text { coverage considered) }\end{array}$ \\
\hline $\begin{array}{l}\text { Height mobile sta- } \\
\text { tion }\end{array}$ & $1.5 \mathrm{~m}$ & $1.5 \mathrm{~m}$ \\
\hline
\end{tabular}
work of Robert MacArthur and Edward Wilson in the early 1960s. Using this model, it was possible to predict the number
TABLE I

LINK BUDGET PARAMETERS FOR THE LTE NETWORK

of species in a habitat. The habitat is an area that is geographically isolated from other habitats. The geographical areas that are well suited as residences for biological species are said to have a high habitat suitability index $(H S I)$. Therefore, every habitat is characterized by the $H S I$ which depends on factors like rainfall, diversity of vegetation, diversity of topographic features, land area, and temperature. Each of the features that characterize habitability is known as suitability index variables (SIV). The SIVs are independent variables while $H S I$ is the dependent variable.

Therefore, a solution to a $D$-dimensional problem can be represented as a vector of SIV variables $\left[S I V_{1}, S I V_{2}, \ldots \ldots \ldots . S I V_{D}\right]$, which is a habitat or island. The value of $H S I$ of a habitat is the value of the objective function that corresponds to that solution and it is found by

$$
H S I=F(\text { habitat })=F\left(S I V_{1}, S I V_{2}, \ldots . . S I V_{D}\right)
$$

Habitats with a high HSI are good solutions of the objective function, while poor solutions are those habitats with a low $H S I$. The immigration and emigration rates are functions of the rank of the given candidate solution. The rank of the given candidate solution represents the number of species in 
a habitat. These are given by

$$
\mu_{k}=E\left(\frac{k}{S_{\max }}\right), \quad \lambda_{k}=I\left(1-\frac{k}{S_{\max }}\right)
$$

where $I$ is the maximum possible immigration rate, $E$ is the maximum possible emigration rate, $k$ is the rank of the given candidate solution, and $S_{\max }$ is the maximum number of species (e.g. population size). The rank of the given candidate solution or the number of species is obtained by sorting the solutions from most fit to least fit, according to the $H S I$ value (e.g. fitness). BBO uses both mutation and migration operators. The application of these operators to each SIV in each solution is decided probabilistically.

\section{A. Opposition Based Learning (OBL)}

The basic concept of OBL was originally introduced by Tizhoosh in [10]. The basic idea of OBL is to calculate the fitness not only of the current individual but also to calculate the fitness of the opposite individual. Then the algorithm selects the individual with the lower (higher) fitness value. At first we give the definitions for the basic concepts of OBL [10]-[12].

Definition (Opposite Number) let $x \in[a, b]$ be any real number. The opposite number is defined by

$$
x_{O}=a+b-x
$$

Definition (Opposite Point). Similarly if we extend the above definition to $D$-dimensional space then let $P\left(x_{1}, x_{2}, \ldots x_{D}\right)$ a point where $x_{1}, x_{2}, \ldots x_{D} \in \Re$ and $x_{j} \in\left[a_{j}, b_{j}\right] \forall j \in\{1,2, \ldots D\}$. The opposite point $P_{O}\left(x_{O 1}, x_{O 2}, \ldots x_{O D}\right)$ is defined by its components

$$
x_{O j}=a_{j}+b_{j}-x_{j}
$$

Definition (Semi-opposite Point) [13]. If we change the components of a point by its opposites only in some components and the other remain unchanged then the new point is a semi-opposite point. This is defined by $P_{S O}\left(x_{S O 1}, x_{S O 2}, . . x_{S O j} . ., x_{S O D}\right)$

$$
\text { where } \forall j \in\{1,2, . ., D\} x_{S O j}=\left\{x_{j} \text { or } x_{O j}\right.
$$

For example in a two-dimensional space where each dimension can be either 0 or 1 we consider the point $P 1(0,1)$. Then the two semi-opposite points are $P 2(0,0)$ and $P 3(1,1)$, while the opposite point is $P 4(1,0)$.

\section{B. Proposed Algorithm}

In this paper we propose a OBBO version based on semiopposite points. We call this algorithm Modified OBBO (MOBBO). We define a new control parameter named opposition probability $p_{o} \in[0,1]$. This parameter controls if a SIV variable in a habitat will be replaced by its opposite or not. Moreover as in previous opposition-based algorithms [7]-[9] we use the jumping rate parameter $j_{r} \in[0,1]$ which controls in each generation if the opposite population is created or not. The opposite based algorithms require two additional parts to the original algorithm code; the opposition-based population initialization and the opposition-based generation jumping [7]-[9]. The opposition based population initialization for MOBBO is described below. For this case $l o w_{j}$, upper $_{j}$ are the lower and upper limits in the $\mathrm{j}$-th dimension respectively.

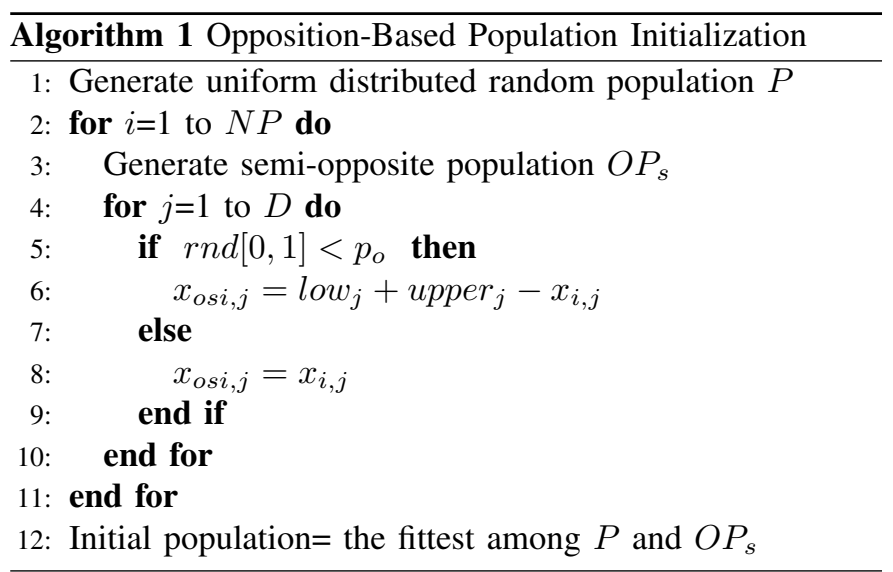

The opposition-based generation jumping follows a similar approach. The algorithm description is given below. The $\min _{j}$, $\max _{j}$ are the minimum and maximum values of the $\mathrm{j}$-th dimension in the current population respectively.

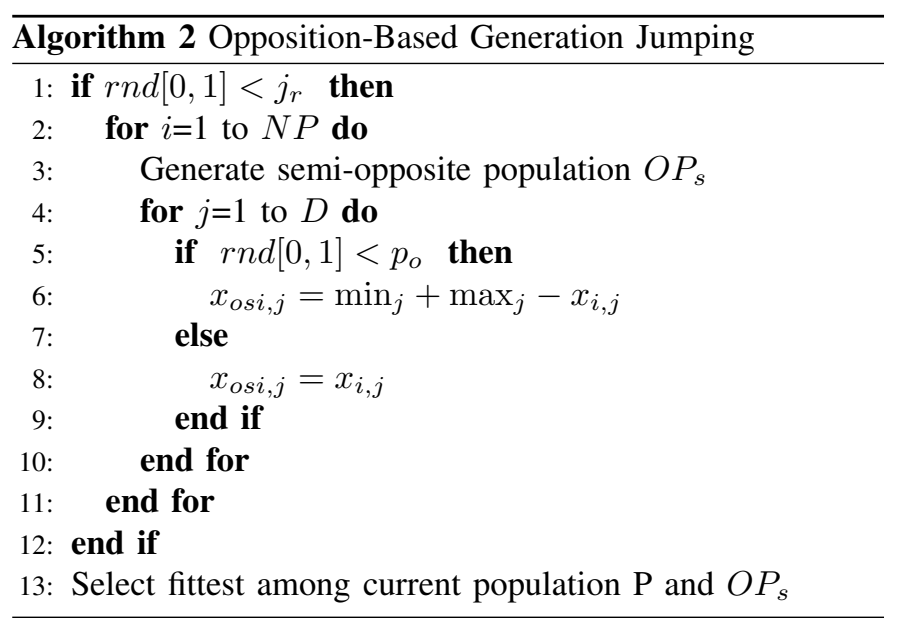

Therefore, the MOBBO algorithm can be described as follows:

1) Initialize the MOBBO control parameters.

2) Initialize a random population of $N P$ habitats (phase vectors) from a uniform distribution. Set the number of generations $G$ to one.

3) Initialize the opposite population according to algorithm 1 .

4) Map the $H S I$ value to the number of species $S$, the immigration rate $\lambda_{k}$, the emigration rate $\mu_{k}$ for each solution (phase vector) of the population.

5) Apply the migration operator for each non-elite habitat based on immigration and emigration rates using (5).

6) Apply the mutation operator.

7) Evaluate objective function value. 


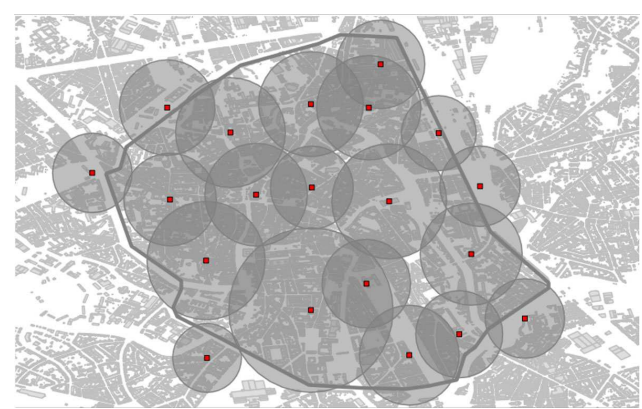

Fig. 1. Map of the city of Ghent with the active LTE base stations for the first case. The circles represent the coverage area of each base station.

8) If $r n d[0,1]<j_{r}$ calculate the opposite population according to algorithm 2 .

9) Repeat step 4 until the maximum number of generations $G_{\max }$ or the maximum number of objective function evaluations is reached.

\section{NumericAl RESUltS}

We consider 75 possible LTE base stations. Each one can be active (1) or not (0). If the base station is active then the range of the input power of the base station antenna is from 0 to $43 \mathrm{dBm}$, and 0 to $33 \mathrm{dBm}$ with a step of $1 \mathrm{dBm}$ for macrocell and femtocell base stations respectively. We compare MOBBO with the original BBO algorithm. Both algorithms are executed 20 times. The results are compared. The population size is set to 100 and the maximum number of generations is set to 1000 iterations. The maximum number of objective function evaluations is set to 100000 . The first case is that of an LTE network with macrocell base stations without Multiple Input Multiple Output (MIMO). The total number of decision variables is $2 \times 75$ for this case (each base station can be active or not and have a value of input power). The bestobtained result for MOBBO is that of a network with about 95\% coverage and $24.5 \%$ power consumption (which means that the power consumption is $24.5 \%$ of the maximum power consumption assuming that all base stations are active and operate at a maximum input power). The solution consists of 20 base stations. This solution is visualized in Fig. 1. Correspondingly, the best-obtained result for the original $\mathrm{BBO}$ is that with $95 \%$ coverage and $25.3 \%$ power consumption, which consists of 21 base stations.

The second case is that of an LTE network supporting both macrocell and femtocell base stations. In this case each base station could be either off (0), macrocell active (1), or femtocell active (2). MOBBO has produced a network that has $95 \%$ coverage and $24.4 \%$ power consumption. The number of base stations in this network is 23 . Fig. 2 visualizes this case. The best result with $\mathrm{BBO}$ is that of a network consisting of 21 base stations with $95 \%$ coverage and $24.7 \%$ power consumption. Both results are very similar in this case.

The final example is that of an LTE network supporting both macrocell and femtocell base stations with MIMO. Thus, each base station has a different number of transmitting and

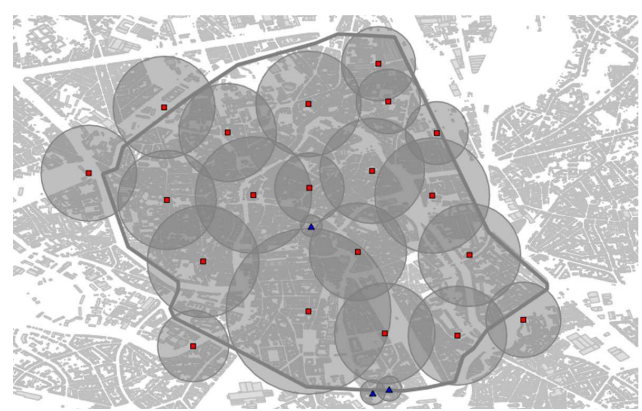

Fig. 2. Map of the city of Ghent with the active LTE base stations for the second case. The circles represent the coverage area of each base station. The red squares indicate the macrocell base stations while the blue triangles indicate the femtocell base stations.

TABLE II

BEST-OBTAINED RESULTS COMPARISON. THE SMALLER VALUES ARE IN BOLD.

\begin{tabular}{|l|c|c|c|}
\hline \multirow{2}{*}{ Algorithm } & \multicolumn{3}{|c|}{ Best objective function value } \\
\cline { 2 - 4 } & Case 1 & Case 2 & Case 3 \\
\hline BBO & -468.64 & -471.62 & -548.97 \\
\hline MOBBO & $\mathbf{- 4 7 2 . 6 5}$ & $\mathbf{- 4 7 3 . 1 2}$ & $\mathbf{- 5 5 0 . 5 2}$ \\
\hline
\end{tabular}

receiving antennas. Each base station could consist of $N_{t}$ transmission and $N_{r}$ reception antennas. The possible values for $N_{t}$ and $N_{r}$ is 1,2 , or 4 . Therefore, the total number of unknowns increases to $4 \times 75$. The best-obtained result for this case using MOBBO is a network with $95 \%$ coverage and $8.9 \%$ power consumption. This solution requires 24 LTE base stations. The network is shown in Fig.3. BBO has obtained a best solution with 21 bases stations with $95 \%$ coverage and $9.2 \%$ power consumption. The best obtained objective function values for each case are shown in Table II. It is obvious that MOBBO has outperformed the original BBO algorithm. Table III reports the average fitness values for different numbers of objective-function evaluations. Again MOBBO outperforms BBO which shows faster convergence.

TABLE III

AVERAGE FITNESS COMPARISON. THE SMALLER VALUES ARE IN BOLD.

\begin{tabular}{|l|l|c|c|c|}
\hline \multirow{2}{*}{ Obj. Func. Evaluations } & \multirow{2}{*}{ Algorithm } & \multicolumn{3}{|c|}{ Average fitness } \\
\cline { 2 - 5 } & & Case 1 & Case 2 & Case 3 \\
\hline \multirow{2}{*}{1000} & BBO & -76.27 & -64.5 & -67.33 \\
\cline { 2 - 5 } & MOBBO & $\mathbf{- 8 0 . 8 5}$ & $\mathbf{- 7 3 . 9 2}$ & $\mathbf{- 7 3 . 3 8}$ \\
\hline \multirow{2}{*}{5000} & BBO & $\mathbf{- 4 1 4 . 2 9}$ & -289.66 & -242.6 \\
\hline \multirow{2}{*}{30000} & MOBBO & -392.78 & $\mathbf{- 4 4 4 . 1 8}$ & $\mathbf{- 5 0 6 . 3}$ \\
\cline { 2 - 5 } & BBO & -456.44 & -450.52 & -536.6 \\
\cline { 2 - 5 } & MOBBO & $\mathbf{- 4 6 3 . 4 8}$ & $\mathbf{- 4 7 1 . 3 3}$ & $\mathbf{- 5 4 3 . 6 5}$ \\
\hline
\end{tabular}

\section{CONCLUSION}

In this paper, we have addressed the problem of designing LTE networks for optimal coverage with the lowest power con- 


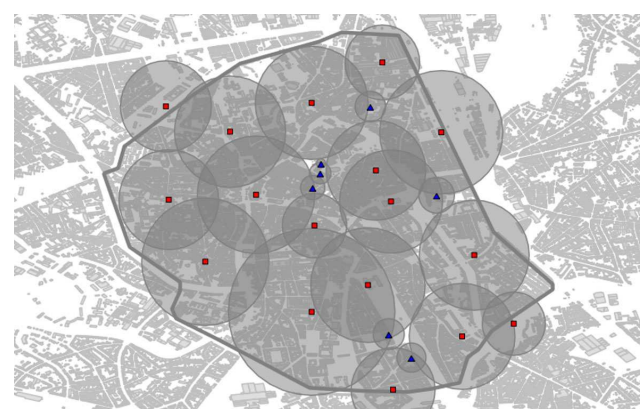

Fig. 3. Map of the city of Ghent with the active LTE base stations for the third case supporting MIMO. The circles represent the coverage area of each base station. The red squares indicate the macrocell base stations while the blue triangles indicate the femtocell base stations.

sumption. We have proposed a novel design approach based on a new Oppositional-based BBO algorithm. The proposed algorithm outperformed the original BBO algorithm in terms of solution accuracy and convergence speed. The numerical results that we have shown have proven the effectiveness of this approach. In our future work, we will study further the capabilities of this new algorithm.

\section{REFERENCES}

[1] W. Van Heddeghem, S. Lambert, B. Lannoo, D. Colle, M. Pickavet, and P. Demeester, "Trends in worldwide ict electricity consumption from 2007 to 2012," Computer Communications, vol. 50, pp. 64-76, 2014.

[2] M. Deruyck, E. Tanghe, W. Joseph, and L. Martens, "Modelling and optimization of power consumption in wireless access networks," Computer Communications, vol. 34, no. 17, pp. 2036-2046, 2011.

[3] M. Deruyck, W. Vereecken, W. Joseph, B. Lannoo, M. Pickavet, and L. Martens, "Reducing the power consumption in wireless access networks: Overview and recommendations," Progress In Electromagnetics Research, vol. 132, pp. 255-274, 2012.

[4] M. Deruyck, W. Joseph, and L. Martens, "Power consumption model for macrocell and microcell base stations," European Transactions on Telecommunications, vol. 25, no. 3, pp. 320-333, 2014.

[5] M. Deruyck, W. Joseph, E. Tanghe, and L. Martens, "Reducing the power consumption in lte-advanced wireless access networks by a capacity based deployment tool," Radio Science, vol. 49, no. 9, pp. 777 787, 2014.

[6] D. Simon, "Biogeography-based optimization," IEEE Transactions on Evolutionary Computation, vol. 12, no. 6, pp. 702-713, 2008.

[7] M. Ergezer, D. Simon, and D. Du, "Oppositional biogeography-based optimization," in Conference Proceedings - IEEE International Conference on Systems, Man and Cybernetics, 2009, pp. 1009-1014.

[8] R. S. Rahnamayan, H. R. Tizhoosh, and M. M. A. Salama, "Oppositionbased differential evolution," IEEE Transactions on Evolutionary Computation, vol. 12, no. 1, pp. 64-79, 2008.

[9] M. Ergezer and D. Simon, "Oppositional biogeography-based optimization for combinatorial problems," in 2011 IEEE Congress of Evolutionary Computation, CEC 2011, 2011, pp. 1496-1503.

[10] H. R. Tizhoosh, "Opposition-based learning: A new scheme for machine intelligence," in Proceedings - International Conference on Computational Intelligence for Modelling, Control and Automation, CIMCA 2005 and International Conference on Intelligent Agents, Web Technologies and Internet, vol. 1, 2005, pp. 695-701.

[11] _ " "Reinforcement learning based on actions and opposite actions," Proceedings of the ICGST International Conference on Artificial Intelligence and Machine Learning, pp. 94-98, 2005.

[12] H. R. Tizhoosh and M. Ventresca, Oppositional Concepts in Computational Intelligence, ser. Oppositional Concepts in Computational Intelligence. New York: Springer, 2008.
[13] F. Mohseni Pour and A. A. Gharaveisi, "Opposition-based discrete action reinforcement learning automata algorithm case study: Optimal design of a pid controller," Turkish Journal of Electrical Engineering and Computer Sciences, vol. 21, no. 6, pp. 1603-1614, 2013. 